\title{
UM FILTRO DE KALMAN ESTENDIDO, COM MATRIZES DE COVARIÂNCIA PRÉ-AJUSTADAS, APLICADAS AO CONTROLE DE VELOCIDADE SENSORLESS DE MOTORES DE INDUÇÃO TRIFÁSICOS
}

\section{ARTIGO ORIGINAL}

LOPES, Leonardo de Magalhães ${ }^{1}$

PEIXOTO, Zélia Myriam Assis ${ }^{2}$

LOPES, Leonardo de Magalhães. PEIXOTO, Zélia Myriam Assis. Um Filtro de Kalman Estendido, com matrizes de covariância pré-ajustadas, aplicadas ao controle de velocidade sensorless de motores de indução trifásicos. Revista Científica Multidisciplinar Núcleo do Conhecimento. Ano 05, Ed. 09, Vol. 02, pp. 109126. Setembro de 2020. ISSN: 2448-0959, Link de acesso: https://www.nucleodoconhecimento.com.br/engenharia-eletrica/filtro-de-

kalman

\section{RESUMO}

Com o surgimento dos métodos de controle sensorless, houve a necessidade da utilização de estimadores e/ou observadores de estados que the conferissem a robustez e precisão necessária no acionamento de motores de indução. Este trabalho trata da aplicação do Filtro de Kalman Estendido (EKF) na estimativa da velocidade e posição do rotor, visando a implementação da técnica de controle vetorial indireto em um sistema de controle de velocidade sensorless para motores de indução trifásicos. O desenvolvimento matemático das variáveis de estado do sistema associado ao processo estocástico do EKF é apresentado neste estudo, e apontam a sua aplicação em condições de velocidade e carga variáveis, as quais são impostas a estes motores

\footnotetext{
${ }^{1}$ Mestre em Engenharia Elétrica e Engenheiro em Eletrônica e Telecomunicação.

${ }^{2}$ Doutora em Engenharia Elétrica.
} 
no cotidiano. A estratégia de controle sensorless foi testada através de linhas de rotina no software Matlab ${ }^{\circledR}$, simulando condições de operação deste tipo de motor, sendo comprovada a sua performance, assim como os tempos de convergência consistentes com os requisitos usuais dos sistemas de alto desempenho. As principais contribuições deste trabalho são o uso de um EKF de ordem reduzida (ROEKF) e o pré-ajuste das matrizes de covariância para agilizar a convergência nas estimativas de velocidade e posição para as futuras implementações em processadores de sinais digitais atualmente acessíveis.

Palavras-chave: EKF, Filtro de Kalman Estendido, Motores de Indução, Sensorless, Sistemas de acionamento elétrico CA.

\section{INTRODUÇÃO}

Nas últimas décadas, as aplicações dos sistemas de acionamento de corrente alternada (CA), associadas as técnicas de controle vetorial com monitoramento da velocidade e/ou posição por meio de sensores eletromecânicos, estabeleceram-se como uma alternativa de baixo custo, maior robustez mecânica e alto desempenho, equiparando-se aos precursores acionamentos CC. Porém, a utilização de transdutores eletromecânicos ainda inclui limitações quanto aos custos de manutenção, confiabilidade, robustez mecânica e restrições quanto a operação em ambientes agressivos (DADKHAH et al., 2015).

Nesse contexto e a partir do surgimento dos processadores digitais de sinais de alta velocidade, os sistemas de controle de velocidade e/ou posição sensorless, embora impliquem no aumento da complexidade matemática e computacional dos modelos de controle, vêm sendo cada vez mais frequentemente utilizados visto contribuírem quanto a robustez mecânica e à variação dos parâmetros das máquinas e incertezas intrínsecas às medições dos sinais elétricos (BARUT et al., 2008).

Diversas pesquisas vêm sendo desenvolvidas visando os sistemas sensorless, onde destacam-se a técnica de modos deslizantes (Sliding Mode), os sistemas adaptativos 
por modelo de referência (Model Reference Adaptive System) e o Filtro de Kalman Estendido (EKF - Extend Kalman Filter), dentre outros (VIEIRA et al., 2014).

Rudolf Emil Kalman apresentou, em 1960, uma solução recursiva para o problema de filtragem linear discreta a partir do modelo no espaço de estados acrescido de parcelas estocásticas referentes aos ruídos de medição e erros de modelagem. Porém, a versão inicial do Filtro de Kalman (KF - Kalman Filter) apresenta limitações em relação a características não-lineares como, no caso dos motores de indução (MI), as não-linearidades referentes à

relação velocidade e fluxos eletromagnéticos bem como a presença de parâmetros variantes no tempo associados a essas variáveis (BARUT et al., 2007).

Uma alternativa ao KF é o Filtro de Kalman Estendido (EKF) cuja estrutura pode ser aplicada a sistemas linearizados em torno de um ponto específico de operação. Outras variantes do Filtro de Kalman encontram-se disponíveis, tais como o Unscented Kalman Filter (UKF) Akin et al. (2003), o EKF Combinado S. Bogosyan and Gokasan (2007), Bi Input-extended Kalman Filter (Bi-EKF), dentre outras.

Em Barut (2010), o UKF é associado ao modelo não-linear do Ml e a um método de filtragem da matriz de covariância. Essa combinação gerou o SRUKF (Square Root UKF), com melhorias na precisão se comparada à versão UKF.

Em Jafarzadeh et al. (2013), é descrita a implementação em tempo real do estimador EKF de ordem completa com entrada dupla ou Bi-EKF. O Bi-EKF é baseado em dois modelos matemáticos, um para o fluxo do estator e o outro para o fluxo do rotor no referencial estacionário, proporcionando a estimativa completa de todos os estados do motor de indução em conjunto com as resistências de estator e rotor, em uma ampla faixa de velocidade (BARUT et al., 2012).

Outro método derivado do EKF é o Filtro de Kalman Estendido de Ordem Reduzida (ROEKF - Reduced-Order Extended Kalman Filter) que, com menor número de variáveis, proporciona a redução do tempo de processamento e dos erros de 
estimação sem comprometer o desempenho do sistema (YIN et al., 2013) (LEITE et al., 2004).

A estimação da velocidade/posição da MI requer o conhecimento das tensões e correntes do estator, porém o número total de variáveis e parâmetros a serem tratados pode se estender até 10, inviabilizando o processamento on line. Vale destacar que a precisão das estimações das variáveis de controle depende fortemente dos parâmetros da MI e da relação sinal-ruído (SNR - Signal Noise Ratio) dos sinais medidos (LEITE et al., 2004).

Este trabalho propõe a aplicação de um Filtro de Kalman Estendido de Ordem Reduzida, especialmente um ROEKF de $5^{\mathrm{a}}$ ordem, para a estimação da velocidade e posição de um motor de indução trifásico com base nas componentes ortogonais da corrente de estator e do fluxo de rotor.

Visando à redução do tempo de processamento e futura implementação utilizando o processador digital de sinais do Fabricante Texas Instrument, modelo TMDSHVMTRPFCKIT, busca-se uma alternativa aos EKFs de $6^{\text {a }}$ e $7^{\text {a }}$ ordens usualmente aplicados que incluem as componentes da corrente de estator, fluxos eletromagnéticos de rotor e as resistências de estator e rotor (DADKHAH et al., 2015) (BOGOSYAN E GOKASAN, 2007) (BARUT, 2010) (BARUT et al., 2012).

As principais contribuições deste trabalho referem-se às análises do ROEKF em relação à precisão e convergência da estimação da velocidade sob diferentes condições de operação e carga bem como a adaptação, desenvolvimento e testes das técnicas de sintonia off line das matrizes de covariância dos erros de modelagem e medições.

\section{REFERENCIAL TEÓRICO}

Nesta seção serão apresentados os conceitos fundamentais relativos ao modelo matemático do MI trifásico, Filtro de Kalman Estendido e técnicas de sintonia off line 
das matrizes de covariância, necessários ao desenvolvimento do ROEKF de $5^{\mathrm{a}}$ ordem para aplicação em um sistema de controle velocidade sensorless (AMEID et al., 2016).

\subsection{O MODELO MATEMÁTICO DO MOTOR DE INDUÇÃO TRIFÁSICO} - MI

O modelo matemático do $\mathrm{Ml}$ pode ser descrito, no referencial estacionário, pelas componentes da corrente de estator e de fluxo do rotor $\phi_{\alpha_{r}}, \phi_{\beta_{r}}$ e velocidade de rotor $\omega_{r}$, conforme as equações 1 a 3 (KRAUSE et al., 2013):

$$
\begin{aligned}
& \left|\begin{array}{c}
i_{\alpha_{s}} \\
i_{\beta_{s}} \\
\phi_{\alpha_{r}} \\
\phi_{\beta_{r}} \\
\omega_{r}
\end{array}\right|=A\left|\begin{array}{c}
i_{\alpha_{s}} \\
i_{\beta_{s}} \\
\phi_{\alpha_{r}} \\
\phi_{\beta_{r}} \\
\omega_{r}
\end{array}\right|+\left|\begin{array}{cc}
\frac{1}{\sigma L_{s}} & 0 \\
0 & 1 \\
0 & \frac{\sigma L_{s}}{0} \\
0 & 0
\end{array}\right|\left|\begin{array}{l}
v_{\alpha_{s}} \\
v_{\beta_{s}}
\end{array}\right| \\
& \left|\begin{array}{l}
i_{\alpha_{s}} \\
i_{\beta_{s}}
\end{array}\right|=\left|\begin{array}{lllll}
1 & 0 & 0 & 0 & 0 \\
0 & 1 & 0 & 0 & 0
\end{array}\right|\left|\begin{array}{c}
i_{\alpha_{s}} \\
i_{\beta_{s}} \\
\phi_{\alpha_{r}} \\
\phi_{\beta_{r}} \\
\omega_{r}
\end{array}\right|
\end{aligned}
$$

onde $a_{1}=\left(\frac{R_{s}}{\sigma L_{s}}+\frac{1-\sigma}{\sigma \tau_{r}}\right), a_{2}=\frac{L_{m}}{\sigma L_{s} L_{r} \tau_{r}}, a_{3}=\frac{L_{m}}{\sigma L_{s} L_{r}}, a_{4}=\frac{L_{m}}{\tau_{r}}, \mathrm{e} a_{5}=\frac{1}{\tau_{r}}$.

\subsection{O FILTRO DE KALMAN ESTENDIDO - EKF}

O EKF baseia-se na versão contínua do Filtro de Kalman, representado pelas seguintes equações lineares discretas Barut (2010): 


$$
x_{(k)}=A_{d} x_{(k-1)}-B_{d} u_{(k-1)}+w_{(k-1)}
$$

Onde $\mathrm{Ad}$ e $\mathrm{Bd}$ são, respectivamente, as matrizes discretas de parâmetros e das entradas do processo, e W contém as parcelas relativas aos erros de modelagem do sistema.

A equação da variável de medição $z(k)$ é dada por:

$$
z_{(k)}=H x_{(k)}+v_{(k)}
$$

Sendo $\mathrm{H}$ a matriz de observação e $\mathrm{v}(\mathrm{k})$, o ruído da medição. Ambos $w(k)$ e $v(k)$ são ruídos brancos com média zero, independentes entre si e com distribuição de probabilidade normal. O estimador de Kalman apresenta duas etapas para estimação, a predição e a correção. Na etapa de predição deve-se prever a projeção do estado no passo a frente ${ }^{\hat{x}_{(k)}^{-}}$, assim como a incerteza na estimação ${ }_{(k)}^{-}$.

$$
\begin{gathered}
\hat{x}_{(k)}^{-}=A_{d} \hat{x}_{(k-1)}-B_{d} u_{(k-1)} \\
P_{(k)}^{-}=A_{d} P_{(k-1)} A_{d}^{T}+Q
\end{gathered}
$$

$P_{(k)}^{-}$é a matriz de covariância do erro do estimador no instante anterior à observação atual, $A_{d}^{T}$ é a transposta da matriz discreta de parâmetros e $\mathrm{Q}$ é a matriz de covariância dos ruídos do processo.

A etapa de correção, necessária para se alcançar o estado estimado ${ }^{\hat{x}_{(k)}}$, é formulada através de uma equação que associa o estado estimado na predição da medida com a diferença entre o valor real medido e o valor predito. Na etapa de correção, tem-se as equações de atualização da medida de ${ }^{\hat{x}_{(k)}^{-}}$bem como da incerteza da estimação $P_{(k)}^{-}$.

$$
\begin{gathered}
\hat{x}_{(k)}=\hat{x}_{(k)}^{-}+K_{(k)}\left(z_{(k)}-H \hat{x}_{(k)}^{-}\right) \\
P_{(k)}=\left(I-K_{(k)} H\right) P_{(k)}^{-}
\end{gathered}
$$


Onde $P(k)$ é a matriz de covariância do erro de estimação atual e I é a matriz identidade.

A diferença $\left(z_{(k)}-H \hat{x}_{(k)}^{-}\right)$indica a divergência entre a medida estimada a priori e a medida atual $\mathrm{z}(\mathrm{k})$. A matriz $\mathrm{K}$, denominada matriz de ganhos do Filtro de Kalman, possibilita minimizar a covariância do erro do processo e é representada por:

$$
K_{(k)}=\frac{P_{(k)}^{-} H^{T}}{H P_{(k)}^{-} H^{T}+R}
$$

De forma recursiva, o processo vai-se repetindo a partir dos resultados estimados a posteriori na busca da predição dos estados a priori.

Com base na versão KF, o Filtro de Kalman Estendido é um observador estocástico que utiliza sinais corrompidos por ruídos indicado para a estimação de variáveis em sistemas não-lineares representados pela função $f$, sendo descrito genericamente conforme as equações (11) e (12):

$$
\begin{gathered}
x_{(k)}=f\left[x_{(k-1)}, u_{(k-1)}, w_{(k-1)}\right] \\
z_{(k)}=h\left[x_{(k)}, v_{(k)}\right]
\end{gathered}
$$

Como os ruídos w(k-1) e v (k) não são conhecidos a cada passo, os vetores de estado e medidas são aproximados, considerando $w(k-1)=0$ e $v(k)=0$ (BARUT et al., 2012):

$$
\begin{gathered}
\hat{x}_{(k)}=f\left[\hat{x}_{(k-1)}, u_{(k-1)}, 0\right] \\
\hat{z}_{(k)}=h\left[\hat{x}_{(k)}, 0\right]
\end{gathered}
$$

As equações linearizadas através das funções $\mathrm{F}$ e $\mathrm{H}$ são matrizes jacobianas calculadas a cada intervalo de tempo. Analogamente às equações do KF, as etapas de predição e atualização das medições do EKF são escritas como:

$$
\begin{gathered}
\hat{x}_{(k)}^{-}=f\left[\hat{x}_{(k-1)}^{-}, u_{(k-1)}, 0\right] \\
P_{(k)}^{-}=F_{(k)} P_{(k-1)} F_{(k)}^{T}+Q_{(k)}
\end{gathered}
$$




$$
\begin{gathered}
\hat{x}_{(k)}=\hat{x}_{(k)}^{-}+K_{(k)}\left[z_{(k)}-H \hat{x}_{(k)}^{-}, 0\right] \\
P_{(k)}=\left(I-K_{(k)} H_{(k)}\right) P_{(k)}^{-}
\end{gathered}
$$

O ganho do filtro é, então, calculado como:

$$
K_{(k)}=\frac{P_{(k)}^{-} H_{(k)}^{T}}{H_{(k)} P_{(k)}^{-} H_{(k)}^{T}+V_{(k)} R_{(k)} V_{(k)}^{T}}
$$

\section{DESENVOLVIMENTO}

\subsection{APLICAÇÃO DO EKF AO CONTROLE DO MOTOR DE INDUÇÃO}

A Figura 1 mostra o sistema de controle de velocidade sensorless associado ao Filtro de Kalman Estendido de Ordem Reduzida. O sistema de controle de velocidade proposto baseia-se na técnica de controle vetorial por orientação indireta de campo (IFOC - Indirect Field Oriented Control), a qual pressupõe o conhecimento da velocidade e posição do rotor para as transformações entre sistemas de eixos de referência, malhas de desacoplamento das tensões de estator e controladores PI utilizados (LI e ZHONG, 2005).

Assim, o vetor de estados a ser estimado pelo ROEKF deverá ser composto como:

$$
\hat{x}_{(k)}=\left[\hat{i}_{\alpha_{s}(k)}, \hat{i}_{\beta_{s}(k)}, \hat{\phi}_{\alpha_{r}(k)}, \hat{\phi}_{\beta_{r}(k)}, \hat{\omega}_{r(k)}\right]^{T}
$$

Onde o ângulo do vetor fluxo magnético do rotor e a velocidade estimada $\hat{\omega}_{r}$ são calculados (EL-HALIM et al., 2012):

$$
\begin{gathered}
\hat{\theta}_{e_{\text {kalman }}}=\arctan \left(\frac{\hat{\phi}_{\beta_{r}}}{\hat{\phi}_{\alpha_{r}}}\right) \\
\hat{\omega}_{r}=\frac{d \hat{\theta}_{e_{\text {kalman }}}}{d t}
\end{gathered}
$$


As principais etapas para a implementação dos sistemas de acionamento sensorless do motor de indução, utilizando o algoritmo ROEKF, são as seguintes (EL-HALIM et al.2012):

- Seleção do modelo discreto de máquina de indução;

- Inicialização das matrizes de covariância Q, P e R;

- Implementação do algoritmo ROEKF.

Figura 1. Controle vetorial sensorless IFOC com estimador de velocidade ROEKF.

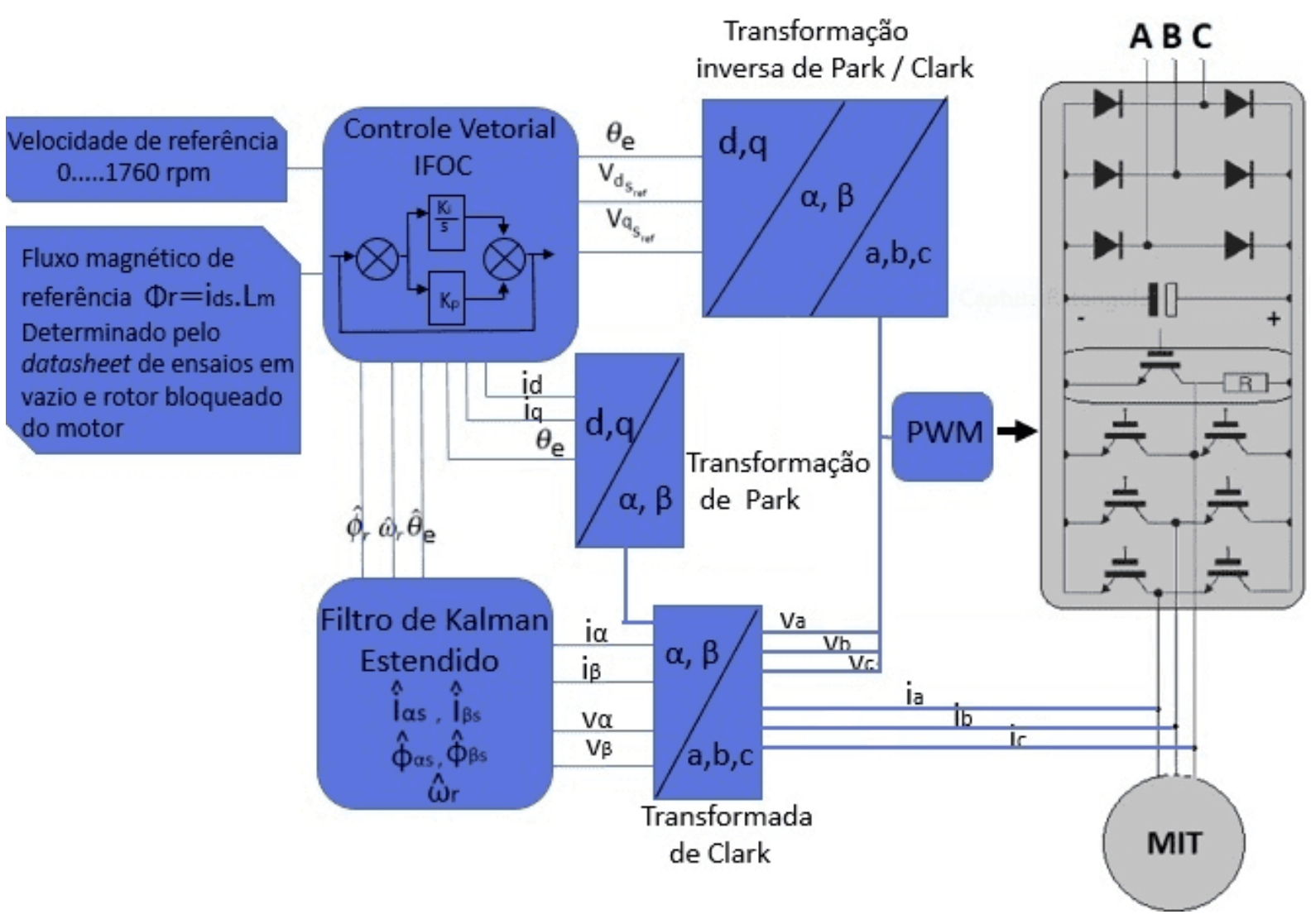

Fonte: O autor adaptou de Shi et al. (2002)

O diagrama da Figura 2 apresenta as etapas de operação do EKF. 
Figura 2. Fluxograma de operação do EKF - Passos da Predição e Correção.

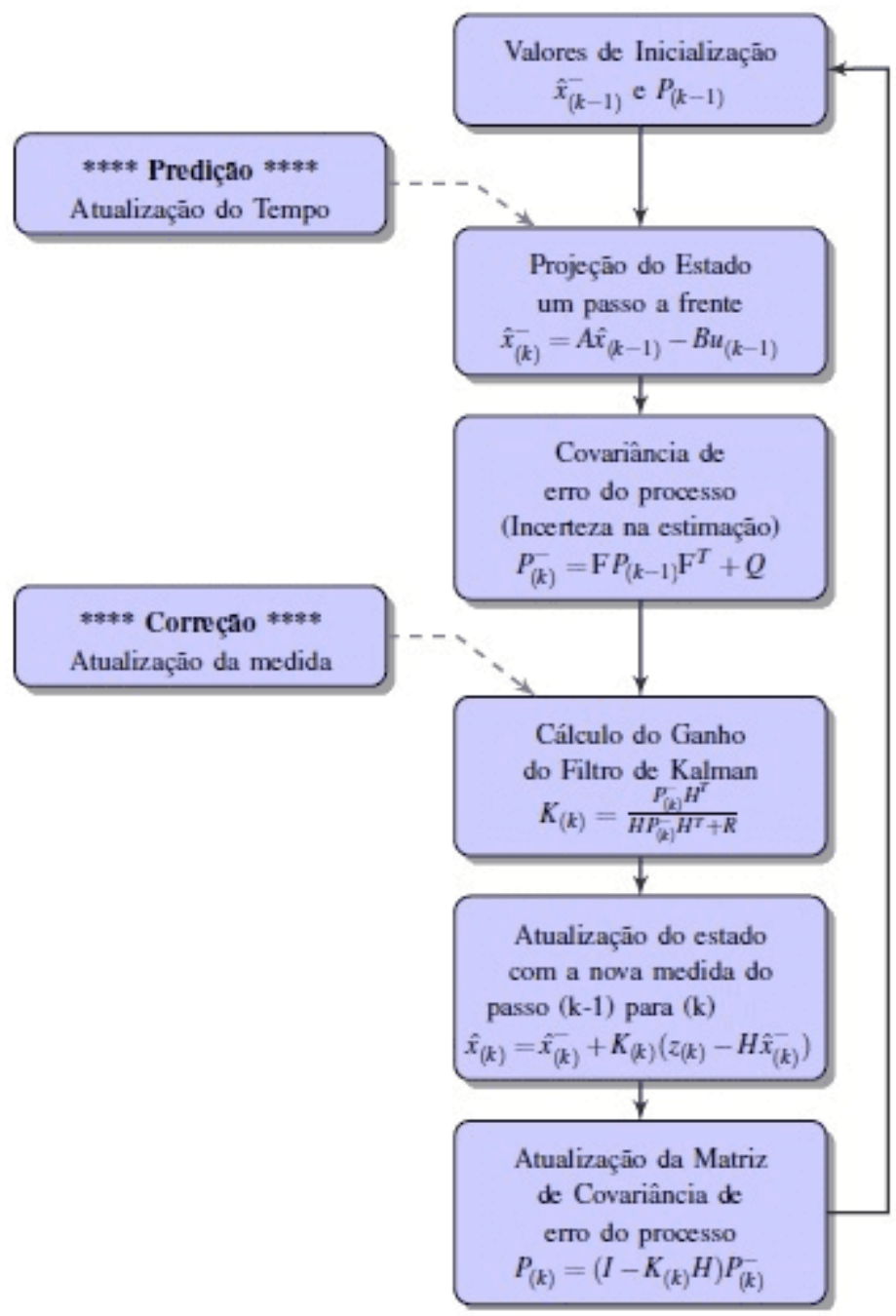

Fonte: O autor adaptou de Leite et al. (2004).

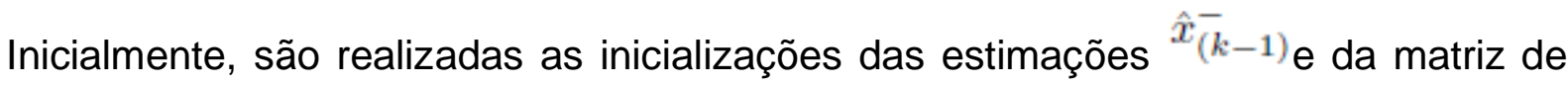
covariância dos erros $\mathrm{P}(\mathrm{k}-1)$. Em seguida, a atualização ou projeção de um passo à frente do estado ${ }^{\hat{x}_{(k)}^{-}}$e da covariância dos erros das estimativas ${ }^{\hat{P}_{(k)}^{-}}$, no estado presente, é obtida a partir da matriz de parâmetros do motor Ad e entradas Bd e u(k1). 
As matrizes de covariância $P, Q$ e $R$, presentes nas etapas do processo, são inicializadas em função da predisposição a incertezas do processo de estimação das variáveis de estado selecionadas.

A correção é feita pela diferença entre o valor medido real e o valor predito. Essa diferença é minimizada pela ação do ganho $K$ sobre a estimativa ${ }^{\hat{x}_{(k)}}$ e sobre a covariância do erro das estimativas do sistema $P(k)$. De forma recursiva, os valores são atualizados, iniciando uma nova estimação.

\subsection{A ESTIMAÇÃO DA VELOCIDADE UTILIZANDO O EKF}

Com base nas equações (15) a (19), a estimação da velocidade da MI será obtida utilizando-se as expressões dadas a seguir.

\subsubsection{ETAPA DE PREDIÇÃO}

$$
F_{(k)}=\left.\frac{\partial}{\partial x}(A x+B u)\right|_{x=\hat{x}_{(k-1)}}
$$

ou seja, $F(k)$ é dado por:

$$
\left|\begin{array}{ccccc}
1-a_{1} T_{s} & 0 & a_{2} T_{s} & a_{3} \hat{\omega}_{r} T_{s} & a_{3} \hat{\phi_{\beta_{r}}} T_{s} \\
0 & 1-a_{1} T_{s} & -a_{3} \hat{\omega}_{r} T_{s} & a_{2} T_{s} & -a_{3} \phi_{\alpha_{r}} T_{s} \\
a_{4} T_{s} & 0 & -a_{5} T_{s} & -\frac{n_{p}}{2} \hat{\omega}_{r} T_{s} & \hat{\phi}_{\beta_{r}} T_{s} \\
0 & a_{4} T_{s} & -\frac{n_{p}}{2} \hat{\omega}_{r} T_{s} & -a_{5} T_{s} & a_{3} \hat{\phi}_{\alpha_{r}} T_{s} \\
0 & 0 & 0 & 0 & 1
\end{array}\right|
$$

\subsubsection{ETAPA DE CORREÇÃO}

$$
H_{d}=\left.\frac{\partial C_{d} x}{\partial x}\right|_{x=x_{(k)}}=\left|\begin{array}{lllll}
1 & 0 & 0 & 0 & 0 \\
0 & 1 & 0 & 0 & 0
\end{array}\right|
$$


A partir das equações (17) e (18), o vetor de estados estimados ${ }^{\hat{x}_{(k)}}$ e as covariâncias dos erros de estimação $\hat{P}_{(k)}$ são corrigidos conforme as expressões:

$$
\begin{gathered}
\hat{x}_{(k)}=\hat{x}_{(k)}^{-}+K_{(k)}\left[z_{(k)}-H_{d(k)} \hat{x}_{(k)}^{-}\right] \\
P_{(k)}=\left(I-K_{(k)} H_{d(k)}\right) P_{(k)}^{-}
\end{gathered}
$$

A cada medição, o filtro armazena as matrizes calculadas no passo anterior e retorna ao início do processo, afim de estimar os próximos valores $\hat{x}_{(k+1)} \mathrm{e}{ }^{\hat{P}_{(k+1)}}$ até que seja obtida a convergência do EKF.

\subsection{SINTONIA OFF LINE DAS MATRIZES DE COVARIÂNCIA P e Q}

O desempenho da estimação é afetado diretamente pelos valores escolhidos para a inicialização das matrizes de covariância $Q, P$ e $R$, na maioria das vezes, baseada em tentativa e erro (RAYYAM et al., 2018). Vários trabalhos vêm sendo desenvolvidos a atender à sintonia dessas matrizes.

Em Zerdali and Barut (2017), os autores utilizam um algoritmo de evolução diferencial (DEA) e um DEA multi objetivo (MODEA) associados a diferentes funções de adequação.

Yin et al. (2017) utiliza uma sequência de múltiplos filtros EKF (MM-EKF), onde diferentes pesos são atribuídos a cada termo do conjunto possibilitando a introdução de novas informações e, em consequência, melhoria de desempenho e redução do erro das estimativas.

Em Wallscheid et al. (2018), é apresentado um projeto conjunto para observação da velocidade, fluxo, torque e estimação dos parâmetros elétricos do MI por meio de um UKF. Embora o UKF tenha mostrado melhor aproximação linear em comparação com o EKF, o estimador UKF apresentou maior sensibilidade aos ruídos das medições on line, levando a uma menor precisão das estimativas de velocidade e torque. 
O método aqui proposto para sintonia off line para a inicialização das matrizes de covariância Q e P foi desenvolvido a partir Estevam et al. (2014), onde a ausência das equações mecânicas no modelo do EKF dificulta a convergência da estimação da velocidade, usualmente obtida por meio do ajuste de ganho do filtro. Os termos da matriz diagonal P5x5, que estão correlacionados com maior expectativa de incertezas na estimação, e os termos da matriz diagonal Q5×5 que estão correlacionados com maior predisposição a ruídos do processo de estimação devem assumir valores elevados, respectivamente para p55 e q55, e valores menores para q11 e q33. As influências de $P$ e $Q$ na estimação da velocidade foi analisada com o sistema de controle operando em malha aberta, ou seja, o EKF não está influenciando na lógica dos controladores PI do controle vetorial.

A sintonia off line das matrizes de covariância de incertezas da estimação, do ruído do sistema e de medida $P, Q$ e $R$, é baseada nos conhecimentos sobre o comportamento dinâmico do motor de indução e a aplicação das ferramentas estatísticas através do valor esperado, média, distribuição de probabilidade gaussiana, variância e covariância. Desta forma são obtidos os valores para os termos das matrizes.

\section{RESULTADOS DA SIMULAÇÃO}

As simulações foram realizadas em ambiente MatLab ${ }^{\circledR}$, considerando o controle em malha aberta e em malha fechada utilizando-se os valores estimados pelo EKF.

Os dados utilizados na simulação, foram do motor modelo 5K33GN2 - Marathon (Regal Beloit Corporations), disponíveis em www.regalbeloit.com/Products/Catalog. Essas características foram adotadas nas simulações, visando à etapa de implementação prática.

O perfil de velocidade incluiu referências em $90 \mathrm{rpm}, 900$ rpm e 1740 rpm, equivalentes a $5 \%, 50 \%$ e $100 \%$ da velocidade nominal do motor de indução, com frequência de chaveamento dos IGBTs em $4 \mathrm{kHz}$, sob condições de operação a vazio e com carga. 
Os valores, estimados a partir do algoritmo EKF, para o conjugado e fluxo eletromagnético, velocidade e posição mecânica do rotor foram aplicados em substituição ás variáveis necessárias ao controle vetorial.

As expressões (28), (29) e (30) indicam as matrizes sintonizadas para os resultados de simulação. Onde:

$$
\begin{gathered}
P=\left|\begin{array}{ccccc}
0.5 & 0 & 0 & 0 & 0 \\
0 & 0.5 & 0 & 0 & 0 \\
0 & 0 & 0.5 & 0 & 0 \\
0 & 0 & 0 & 0.5 & 0 \\
0 & 0 & 0 & 0 & 50000
\end{array}\right| \\
Q=\left|\begin{array}{ccccc}
0.000024 & 0 & 0 & 0 & 0 \\
0 & 0.00024 & 0 & 0 & 0 \\
0 & 0 & 0.000024 & 0 & 0 \\
0 & 0 & 0 & 0.00024 & 0 \\
0 & 0 & 0 & 0 & 240000
\end{array}\right| \\
R=\left|\begin{array}{cc}
2.4 & 0 \\
0 & 2.4
\end{array}\right|
\end{gathered}
$$

O gráfico mostrado na Figura 3 atesta a convergência do EKF quanto a precisão na estimação, seja em velocidades próximas de zero, velocidades intermediárias ou velocidade nominal. A estimação apresenta valores próximos a velocidade de referência e a velocidade calculada pelo modelo matemático do motor de indução. 
Figura 3. Velocidade variável (90rpm, 900rpm e 1740 rpm).

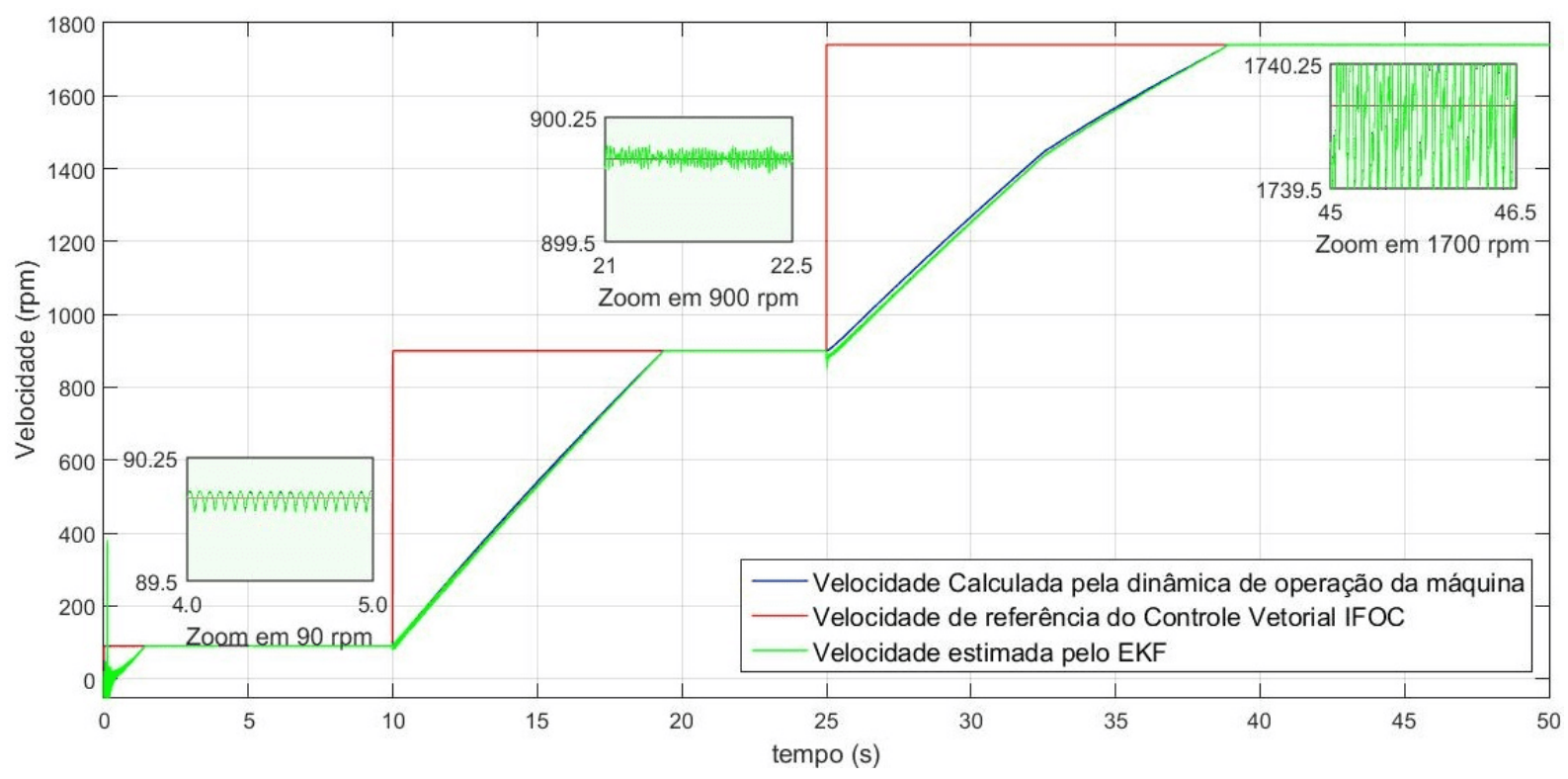

Fonte: O próprio autor

O gráfico da Figura 4 mostra a resposta dinâmica do EKF quanto a posição mecânica do rotor, com convergência na estimação para valores próximos à posição mecânica calculada pelo modelo matemático do motor de indução.

Figura 4. Posição em radianos no tempo $(-\pi, 0, \pi)$.

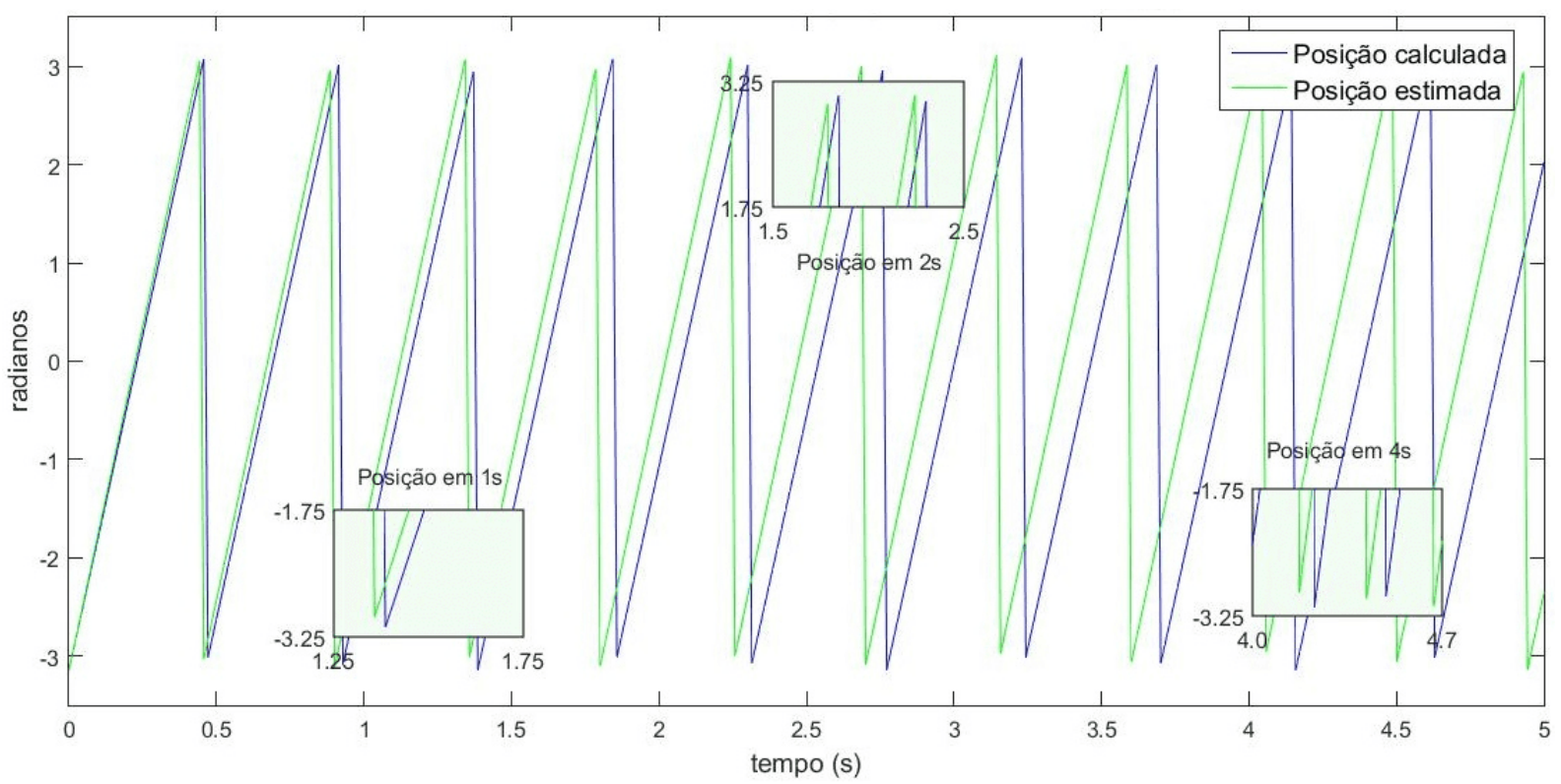

Fonte: O próprio autor

RC: 59207

Disponível em: https://www.nucleodoconhecimento.com.br/engenharia-eletrica/filtro-de-kalman 
Tomando-se a medição da posição em radianos no tempo, com trajetória variando de - ח а $\pi$, o estimador EKF demonstra precisão e convergência em velocidades menores, por exemplo $90 \mathrm{rpm}$, bem como em velocidades médias. Constata-se desvios a partir do instante 25 segundos na Figura 3, onde a velocidade é comutada para 1740 rpm. Nesse caso, os desvios devem-se a lógica de atualização das estimações, as quais estão diretamente ligadas à matriz $\mathrm{P}$ de covariância dos erros da estimação. As incertezas das estimações apresentam evidências devido às iterações dos valores tratados pela fase de predição e, posteriormente, correção das estimações.

O gráfico mostrado na Figura 5 esboça a variação da posição mecânica do rotor em graus.

Figura 5. Posição em graus $0^{\circ} \ldots 360^{\circ}$ variando com a velocidade de 90rpm, 900rpm, e $1740 \mathrm{rpm}$.



graus mecânicos

Fonte: O próprio autor 
Onde:

$$
\frac{d \theta_{m e c}}{d t}=\omega_{r}
$$

Observa-se, porém, a presença de oscilações em torno dos valores de referência. Essas oscilações podem ser minimizadas por meio do ajuste e sintonia do EKF, basicamente sobre as matrizes de covariância $Q, R, P$ e ganho K do EKF. As maiores oscilações destacam-se na operação em velocidades baixas e transitórios.

Quando a matriz P de covariância do erro do estimador dos estados se aproxima de zero, a matriz $\mathrm{K}$ de ganho atua menos intensamente para a minimização da discrepância entre os valores de predição e as medidas tomadas sobre o modelo da máquina.

Caso sejam adotados valores mais elevados para os elementos da matriz de covariância dos ruídos $(\mathrm{Q})$ há um maior grau de incerteza em relação às estimações dos estados do sistema, implicando no incremento da matriz do ganho $\mathrm{K}$ e aumento na velocidade de convergência da estimação.

O aumento nos valores dos elementos da matriz de covariância dos ruídos de medição das correntes e tensões, $\mathrm{R}$, implica na diminuição da matriz do ganho $\mathrm{K}$, a qual atua na redução da velocidade de resposta do EKF.

\section{CONCLUSÕES}

Os resultados simulados apontam a viabilidade do estimador com base nos indicativos de sua capacidade para resposta às variações da velocidade impostas ao sistema de controle, assim como diferentes condições de carga impostas ao motor.

Foram simuladas condições de operação do motor de indução com diferentes cargas, até $400 \mathrm{~N}$, ou aproximadamente $40 \mathrm{~kg}$ de peso. Os valores de carregamento estão baseados na capacidade do Torque mecânico do motor, conforme o datasheet do fabricante, sendo 1,1 N.m com velocidade nominal de 1725 rpm. 
A estimação do EKF apresenta convergência com os valores de referência e valores calculados, de acordo com a dinâmica de operação imposta a cada condição de carga.

De posse dos resultados apresentados, espera-se implementar a solução em um hardware para controle digital de motores utilizando-se o processador digital de sinais do Fabricante Texas Instrument, modelo TMDSHVMTRPFCKIT com placa de controle F28035 e um motor de indução trifásico assíncrono do tipo gaiola de esquilo, modelo 5K33GN2, do fabricante Marathon (Regal Beloit Corporations), disponível no PPGEE - PUCMINAS.

\section{REFERÊNCIAS}

AKIN, B., ORGUNER, U., and ERSAK, A. (2003). State estimation of induction motor using unscented Kalman Filter. IEEE Conference on Control Applications, 915-919.

AMEID, T., MENACER, A., TALHAOUI, H., HARZELLI, I., and AMMAR, A. (2016). Simulation and real-time implementation of sensorless field oriented control of induction motor at healthy state using rotor cage model and ekf. $8^{\text {th }}$ International Conference on Modelling, Identification and Control, 695-700.

BARUT, M. (2010). Bi input-extended Kalman Filter based estimation technique for speed-sensorless control of induction motors. Energy Conversion and Management, 2032-2040.

BARUT, M., BOGOSYAN, S., and GOKASAN, M. (2007). Speed sensorless estimation for induction motors using extended Kalman Filters. IEEE Transactions on Industrial Electronics, 272-280.

BARUT, M., BOGOSYAN, S., and GOKASAN, M. (2008). Experimental evaluation of braided ekf for sensorless control of induction motors. IEEE Transactions on Industrial Electronics, 620-632. 
BARUT, M., DEMIR, R., ZERDALI, E., and INAN, R. (2012). Real-time implementation of bi input-extended Kalman Filter-based estimator for speedsensorless control of induction motors. IEEE Transactions on Industrial Electronics, 4197-4206.

DADKHAH, R., GIVI, H., and MEHDIPOUR, A. (2015). Parameter estimation of the induction motor using extended Kalman Filter for wide range speed control. The $6^{\text {th }}$ International Power Electronics Drive Systems and Technologies Conference (PEDSTC2015), 137-142.

EL-HALIM, A.F.A., ABDULLA, M.M., and EL-ARABAWY, I.F. (2012). Simulation aides in comparison between different methodology of field oriented control of induction motor based on flux and speed estimation. $22^{\text {nd }}$ International Conference on Computer Theory and Applications (ICCTA), 117-121.

ESTEVAM, E.G.A., PEIXOTO, Z.M.A., and FERREIRA, F.M.F. (2014). Aplicação EKF e as técnicas DTC-MTPA ao controle de velocidade sensorless do ipmsm. 20ํㅡㄹ Congresso Brasileiro de Automática, 1348-1355.

JAFARZADEH, S., LASCU, C., and FADALI, M.S. (2013). Square root unscented Kalman Filters for state estimation of induction motor drives. IEEE Transaction on Industry Applications, 92-99.

KRAUSE, P., WASYNCZUK, O., SUDHOFF, S., and PEKAREK, S. (2013). Analysis of Electric Machinery and Drive Systems. Wiley-IEEE Press, New Jersey.

LEITE, A.V., ARAUJO, R.E., and FREITAS, D. (2004). Full and reduced order extended Kalman Filter for speed estimation in induction motor drives: A comparative study. $35^{\text {th }}$ Annual IEEE Power Electronics Specialists Conference, 2293-2299.

LI, J. and ZHONG, Y. (2005). Comparison of three Kalman Filters for speed estimation of induction machines. $40^{\text {th }}$ Annual Meeting of the IEEE Industry Applications Society, 1792-1797. 
RAYYAM, M., ZAZI, M., and BARRADI, Y. (2018). A new metaheuristic unscented Kalman Filter for state vector estimation of the induction motor based on ant lion optimizer. COMPEL - The international journal for computation and mathematics in electrical and electronic engineering, 1054-1068.

S. BOGOSYAN, M.B. and GOKASAN, M. (2007). Braided extended Kalman Filters for sensorless estimation in induction motors at high-low/zero speed. IET Control Theory and Applications, 987-998.

SHI, K.L., CHAN, T.F., WONG, Y.K., and HO, S.L. (2002). Speed estimation of an induction motor drive using an optimized extended Kalman Filter. IEEE Transactions on Industrial Electronics, 124-133.

VIEIRA, R., GASTALDINI, C.C., AZZOLIN, R.Z., and GRUNDLING, H.A. (2014). Sensorless sliding-mode rotor speed observer of induction machines based on magnetizing current estimation. IEEE Transactions on Industrial Electronics, 45734582.

WALLSCHEID, O., SCHENKE, M., and BÖCKER, J. (2018). Improving torque and speed estimation accuracy by conjoint parameter identification and unscented Kalman Filter design for induction machines. International Conference on Electrical Machines and Systems ICEMS, 1181-1186.

YIN, Z., LI, G., ZHANG, Y., LIU, J., SUN, X., and ZHONG, Y. (2017). Speed and flux observer of induction motor based on extended Kalman Filter and Markov chain. IEEE Transactions on Power Electronics, 7096-7117.

YIN, Z., ZHAO, C., LIU, J., and ZHONG, Y. (2013). Research on anti-error performance of speed and flux estimator for induction motor using robust Reduced-Order EKF. IEEE Transactions on Industrial Informatics, 1037-1046.

ZERDALI, E. and BARUT, M. (2017). The comparisons of optimized extended Kalman Filters for speed-sensorless control of induction motors. IEEE Transactions on Industrial Electronics, 4340-4351. 
Enviado: Agosto, 2020.

Aprovado: Setembro, 2020. 